\title{
Expectativas y percepciones de docentes y directivos docentes sobre las políticas educativas y los procesos formativos en los programas de formación de maestros en Norte de Santander
}

\section{Expectations and perceptions of teachers and teacher managers on educational policies and training processes in teacher training programs in Norte de Santander}

\author{
Audin Aloiso Gamboa Suárez, \\ César Augusto Hernández Suárez², \\ William Rodrigo Avendaño Castro ${ }^{3}$
}

\section{RESUMEN}

El artículo pretende mostrar las expectativas y percepciones que tienen docentes y directivos

1 Doctor en Ciencias de la Educación por la Universidad de Cartagena (Colombia). Docente investigador de la Universidad Francisco de Paula Santander. Correo electrónico: audingamboa@ufps.edu.co. Orcid: 0000-00019755-6408.

2 Magister en Educación Matemática por la Universidad Nacional Experimental del Táchira (Venezuela). Docente investigador de la Universidad Francisco de Paula Santander. Correo electrónico: cesaraugusto@ufps.edu.co. Orcid: 0000-0002-7974-5560.

$3 \quad$ Doctor en Ciencias Sociales y Humanas por la Pontificia Universidad Javeriana. Docente investigador de la Universidad Francisco de Paula Santander. Correo electrónico: williamavendano@ufps.edu.co. Orcid: 0000-00027510-8222. docentes sobre las políticas educativas y los procesos formativos que han permeado los currículos y las intenciones académicas de los programas de formación de maestros en Colombia. El estudio se enmarca en el enfoque cuantitativo - descriptivo. Se aplicó una encuesta tipo Likert a 83 docentes y directivos docentes de 23 instituciones educativas de cinco municipios en Norte de Santander. Los resultados evidencian que las percepciones y expectativas de los actores educativos se centran en la necesidad de fortalecer y armonizar el saber pedagógico con el saber disciplinar y dar nuevas posibilidades a los maestros en formación para 
que interactúen con los contextos de práctica pedagógica de una forma más permanente y con mayor rigor educativo.

\section{PALABRAS CLAVE:}

expectativas; políticas educativas; procesos formativos; percepciones

\section{ABSTRACT}

The article aims to show the expectations and perceptions that teachers and teacher managers have on the educational policies and training processes that have permeated the curricula and the academic intentions of teacher training programs in Colombia. The study is framed in the quantitative - descriptive approach. A lickertype survey was applied to 83 teachers and teaching directors of 23 educational institutions of five municipalities in Norte de Santander. The results show that the perceptions and expectations of educational actors focus on the need to strengthen and harmonize pedagogical knowledge with disciplinary knowledge and give new possibilities to teachers in training to interact with the contexts of pedagogical practice in a more permanent and with greater educational rigor.

\section{KEYWORDS:}

expectations; educational policies; training processes; perceptions

\section{INTRODUCCIÓN}

Los programas de formación de maestros en Colombia se remontan a la creación de las escuelas normales en 1982, las cuales tomaron la responsabilidad de preparar a los futuros docentes para educar a los niños de la época. Báez (2005), afirma que en la época eran muy pocos los maestros formados para enfrentar estos desafíos educativos, y por esta razón el gobierno nacional dictó el decreto de 2 de noviembre de 1844, mediante el cual se ordenó el establecimiento de las escuelas normales en cada capital de provincia de la república. Sobre esta base se constituye la escuela normal de Bogotá, que logró organizarse con capital mixto, es decir, de particulares y del gobierno. Esta institución que prácticamente fue la única que funcionó en Nueva Granada, tuvo vigencia por unos veinte años y luego desapareció (p. 429)

Estos antecedentes dieron origen a las primeras facultades de educación en Colombia, las cuales surgieron como una unión entre instituciones ya fundadas como el Instituto Pedagógico Femenino en Bogotá y la Escuela Normal de Varones de Tunja y con las llamadas contribuciones de las Misiones alemanas, y desde el Gimnasio Moderno, en 1914 logrando configurar en Colombia lo que se denominó las institucionalización de las prácticas de saber (Figueroa, 2006).

En este sentido, son diversas las investigaciones y el origen y evolución de los programas de formación de maestros en Colombia. Estos estudios se han centrado principalmente en las escuelas normales y las facultades de educación, en la primera se analizan elementos como la circulación del saber pedagógico (Geles y Ferrerira, 2018; Beltrán, 2018), reformas educativas (Garcés, 1994; Rátova, 2018), orientación vocacional de los normalistas (Navarrete, 2018) y la calidad de la educación en las escuelas normales (Montes, Romero y Gamboa, 2017). Con respecto a las facultades de educación son aún más prolíficos los estudios y se relacionan directamente con la historicidad en la formación de maestros en Colombia (Herrera, 1993), debates teóricos sobre las ciencias de la educación desde las facultades de educación (Castro, 2000; Betancourt y Tovar, 2012) y el impacto de las facultades a 
las transformaciones educativas en Colombia (Cavadias, 2014; Gamboa, 2016), entre otros.

Es en este último aspecto donde el sistema de aseguramiento de la calidad colombiano ha puesto su mirada para aumentar la exigencia en temas de excelencia, iniciativa que surge con las normas para los programas de formación de maestros: desde la ley 115 de 1994, la Acreditación Previa en 1998; El Decreto 272 de 1998 que recogió y reconoció los núcleos básicos y comunes del saber pedagógico; los ECAES (Exámenes de Estado de la Calidad de la Educación Superior) en el 2001, hoy Saber Pro; El Decreto 1278 de 2002 (Estatuto de Profesionalización Docente), los lineamientos para el otorgamiento y la renovación del registro calificado de los programas de formación inicial y permanente de maestros (2002 - 2013); la consolidación de los procesos de autoevaluación y acreditación de programas e instituciones; la elaboración del documento Sistema Colombiano de Formación de Educadores y Lineamientos de Política (2013) y la Resolución 18583 de 2017 por la cual se ajustan las características específicas de calidad de los programas de Licenciatura para la obtención, renovación o modificación del registro calificado y se deroga la Resolución 2041 de 2016 hasta el fallido decreto 1280 del 2018 y hoy el decreto 1330 , entre otros.

Este panorama normativo ha generado diferentes reacciones en actores educativos. Estudios como los de Arias et al., (2018) afirman que la exigencia sobre la acreditación generó grandes premuras a las universidades y se hace cuestionable las exigencias gubernamentales $y$ la igualdad de condiciones con las que se mide la acreditación de programas académicos en Colombia.

Por estos argumentos, se hace necesario indagar sobre las percepciones que tienen docentes y directivos docentes sobre las políticas educativas y los procesos de formación de maestros en Colombia desde su experiencia en lógicas y dinámicas escolares.

\section{METODOLOGÍA}

Este trabajo investigativo se enmarca dentro de las características del enfoque cuantitativo puesto que los datos fueron recolectados en un momento específico de tiempo (primera semana de febrero del 2021) para a partir de ellos determinar frecuencias y porcentajes, por lo que corresponde a las particularidades del nivel descriptivo transversal. En un trabajo preliminar a esta publicación se realiza la validación de la escala y se logra verificar su validez de contenido de cada uno de los ítems mediante el juicio de expertos y posteriormente con el cálculo del estadístico Alfa de Cronbach cuyo valor se ubica en .898 que en opinión de George y Mallery (2003) se puede concluir que el cuestionario es fiable. En lo que respecta al diseño adoptado para la recolección de datos, se ajusta al diseño de campo porque debido a la actual declaratorio de pandemia por causa del Covid-19, se evitan al máximo los contactos físicos entre personas, por lo cual el cuestionario fue diseñado mediante Google Form y fue auto diligenciado por medio de la conexión a internet, por cada uno de los participantes.

Creado el cuestionario fue enviado a la cuenta de correo de 123 docente y directivos que laboraban en diversos niveles educativos en distintas instituciones de educación con sede en el Departamento Norte de Santander. Del grupo de convocados se obtuvo respuesta de 83 personas quienes diligenciaron completamente el cuestionario durante la ventana de tiempo habilitada para tal fin.

En lo que respecta a las características demográficas de los participantes se puede afirmar que:

a) En cuanto al sexo, el $50.8 \%$ son hombres y el porcentaje restante corresponden 
a las mujeres, luego se identificó un ligero predominio del género masculino en la muestra.

b) Respecto a la edad se generaron varios intervalos dentro de lo que se destaca, el $46.4 \%$ de ellos tienes edades que oscilan entre 30 y 40 años, mientras que el $36.1 \%$ tiene edades que van entre 41 y 50 años, finalmente en el $17.5 \%$ restante se observaron que tenían 51 años o más.

c) En cuanto al municipio de residencia, se determinó que el $64.0 \%$ vivían en la ciudad de Cúcuta y su área metropolitana, mientras que el porcentaje restante corresponde las ciudades de Ocaña y Pamplona, ambas ubicadas fuera de la capital pero que cuentan con centros educativos tanto de básica, media y superior.

d) El 55.8\% laboran en instituciones de Educación Superior algunas de orden público y otras privadas, mientras que el porcentaje restante ejercen su actividad laboral en diversas instituciones educativas que ofrecen todos los grados de la Educación Básica y Media.

e) En lo que respecta al tipo de actividad que desempeñan a interior de las instituciones educativas en dónde laboran, se determinó que el $78.4 \%$ de los participantes son docentes de tiempo completo, mientras que el porcentaje restante desempeñan funciones académicoadministrativas bien sea como Coordinadores Académicos o Rectores en caso de instituciones de básica y media en el $15.6 \%$, mientras que en la educación superior han asumido funciones de Coordinador de programa académico y unos pocos, como Decanos.

f) Finalmente, en lo que respecta a la formación académica de los participantes, se pudo determinar que el $19.4 \%$ cuentan sólo con su título de pregrado que en su mayoría son Licenciados en diversas áreas; mientras que el porcentaje restante ha cursado algún nivel de posgrado, observando que el $46.9 \%$ cuentan con título de Magister, el $21.7 \%$ con Especialización y el $12.0 \%$ estudios de Doctorado o Postdoctorado.

\section{RESULTADOS Y DISCUSIÓN}

Los 21 ítems considerados en el instrumento fueron evaluados por cada uno de los participantes mediante el uso de una escala Likert con cinco niveles distribuidos así: dos niveles de postura de rechazo (Totalmente en desacuerdo y En desacuerdo), un punto intermedio que se representa mediante una postura Indiferente y dos niveles de aceptación (De acuerdo y Totalmente de acuerdo). Por simplicidad en la interpretación de los datos, se proceden a reportan en los resultados sólo los tres niveles de postura (De rechazo, Indiferente y De aceptación). La totalidad de ítems se distribuyeron en cinco dimensiones tal como se muestra en la Tabla 1.

Tabla 1. Distribución de los ítems por dimensión de análisis

\begin{tabular}{lc}
\hline \multicolumn{1}{c}{ Dimensión } & $\mathbf{N}^{\circ}$ de ítems \\
\hline Políticas Educativas & 12 \\
Procesos de & 2 \\
Acreditación & \\
Globalización & 3 \\
Procesos Formativos & 2 \\
Formación Docente & 2 \\
\hline
\end{tabular}

Como se puede observar en la Tabla 1 el $57.4 \%$ de los ítems del cuestionario se concentran en la dimensión de las Políticas Educativas dada la importancia de esta dimensión, pues ella tiene impacto directo en las otras cuatro dimensiones.

En la Figura 1 se muestra el comparativo de percepción de opiniones de los informantes en cada una de las dimensiones. Se pudo determinar que las dimensiones con mejor percepción son los Procesos Formativos y de Acreditación, mientras que las dimensiones con peor percepción corresponden a las dimensiones 
de Políticas Educativas y Formación Docente. Ya desde una visión general, el $76 \%$ de los participantes asumieron una postura de aceptación y apoyo ante cada uno de los ítems evaluados en el cuestionario.

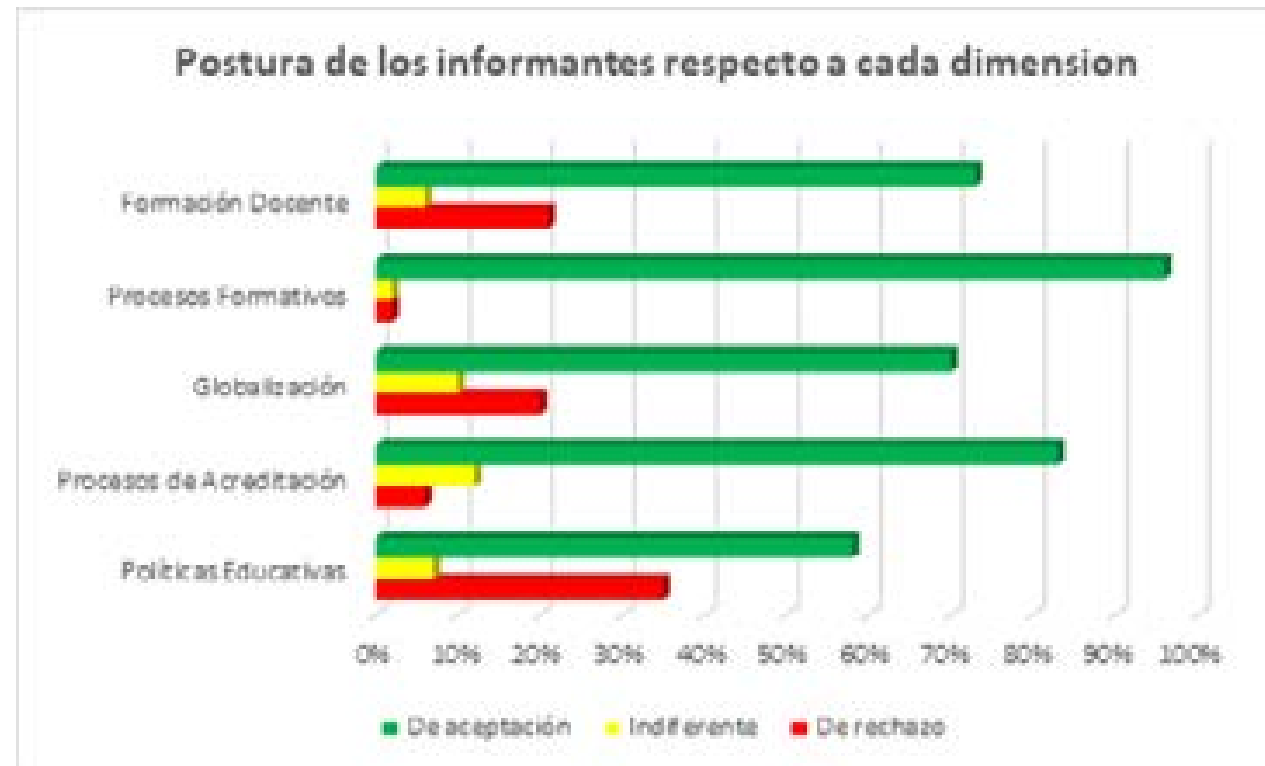

Figura 1. Postura promedio de los informantes respecto a cada una de las dimensiones evaluadas

A continuación, se analizan en detalle cada una de las dimensiones. Con respecto a la dimensión Políticas Educativas (ver Tabla 1), se determinó que a nivel general el $58 \%$ de los informantes manifestaron una postura de aprobación ante cada uno de los ítems pertenecientes a esta dimensión. Se destacan que al menos el $90 \%$ de las participantes manifiestan estar a favor de que es necesario que el sistema escolar se adapte y transforme acorde con la diversidad territorial y poblacional de nuestro país, que responda y se articule con las transformaciones de los campos científicos y humanistas contemporáneos, que sea visto desde el gobierno como un elemento esencial para la construcción de nación y que las políticas educativas no sean organizadas como un proyecto a largo plazo que sea respetado independientemente del gobierno de turno.
Así mismo, los participantes se mostraron inconformes con el cumplimiento del Estado en cuanto a lo que esperan de la Educación en general, desde los indicadores de cobertura, calidad y coherencia académica. Ratifican que es necesario separar la política del proceso educativo para avanzar en la construcción de un mejor país. 
Tabla 1. Indicadores de la Dimensión Políticas Educativas

\begin{tabular}{|c|c|c|c|}
\hline \multirow[b]{2}{*}{ Items } & \multicolumn{3}{|c|}{ Postura } \\
\hline & $\begin{array}{c}\text { De } \\
\text { rechazo }\end{array}$ & Indiferente & $\begin{array}{c}\text { De } \\
\text { aceptación }\end{array}$ \\
\hline $\begin{array}{l}\text { Considera que la legislación colombiana valora con } \\
\text { suficiencia la labor del docente y propone políticas que } \\
\text { favorecen el desarrollo de la educación y el sistema } \\
\text { educativo de acuerdo a retos y necesidades de la población. }\end{array}$ & $74 \%$ & $14 \%$ & $12 \%$ \\
\hline $\begin{array}{l}\text { Las instituciones del Estado actúan de forma lógica, } \\
\text { articulada y coherente en la aplicación de las normas } \\
\text { relativas a la educación. }\end{array}$ & $68 \%$ & $9 \%$ & $23 \%$ \\
\hline $\begin{array}{l}\text { Considera que una de las razones de la crisis del sector } \\
\text { educativo en Colombia es la ausencia de continuidad en las } \\
\text { políticas públicas en educación. }\end{array}$ & $3 \%$ & $6 \%$ & $91 \%$ \\
\hline Considera que los indicadores de cobertura en Educación & & & \\
\hline $\begin{array}{l}\text { Superior son suficiente para evidenciar el cumplimiento de } \\
\text { las políticas educativas colombianas. }\end{array}$ & $85 \%$ & $6 \%$ & $9 \%$ \\
\hline $\begin{array}{l}\text { La autonomía y libertad de cátedra son principios básicos } \\
\text { para la formación de docentes y para el sistema educativo } \\
\text { colombiano. }\end{array}$ & $9 \%$ & $5 \%$ & $86 \%$ \\
\hline $\begin{array}{l}\text { El estado cumple con las expectativas ciudadanas en } \\
\text { cuanto a las decisiones sobre la educación en Colombia. }\end{array}$ & $88 \%$ & $9 \%$ & $3 \%$ \\
\hline $\begin{array}{l}\text { Considera que Colombia debería apropiarse de las metas } \\
\text { del milenio como hoja de ruta para guiar las políticas } \\
\text { educativas, entendidas como política de Estado. }\end{array}$ & $5 \%$ & $10 \%$ & $85 \%$ \\
\hline $\begin{array}{l}\text { El sistema educativo se percibe desde el Estado como un } \\
\text { indicador de logros gubernamentales más que como un } \\
\text { sistema esencial para la construcción de nación. }\end{array}$ & $5 \%$ & $2 \%$ & $93 \%$ \\
\hline $\begin{array}{l}\text { Hay coherencia y articulación entre lo planteado por los } \\
\text { intelectuales de la educación y las acciones del Legislativo y } \\
\text { el Ejecutivo frente a la educación. }\end{array}$ & $82 \%$ & $6 \%$ & $12 \%$ \\
\hline $\begin{array}{l}\text { Es necesario que el sistema escolar colombiano responda y } \\
\text { se articule a los debates y transformaciones de los campos } \\
\text { científicos y humanistas contemporáneos. }\end{array}$ & $0 \%$ & $1 \%$ & $99 \%$ \\
\hline $\begin{array}{l}\text { Es necesario que el sistema escolar colombiano se adapte y } \\
\text { transforme acorde con la diversidad territorial y poblacional } \\
\text { del país. }\end{array}$ & $0 \%$ & $0 \%$ & $100 \%$ \\
\hline $\begin{array}{l}\text { El sistema escolar de una nación multiétnica debería } \\
\text { validar formas de conocimiento diferentes al conocimiento } \\
\text { científico. }\end{array}$ & $2 \%$ & $12 \%$ & $86 \%$ \\
\hline Porcentaje Promedio & $35 \%$ & $7 \%$ & $58 \%$ \\
\hline
\end{tabular}


Por medio de la Tabla 2 se puede evidenciar que a nivel general el $83 \%$ de los participantes tienen una percepción de aprobación respecto a los aspectos pertenecientes a la dimensión de Procesos de Acreditación. Reconocen como fortaleza la función de control que garantiza la calidad que se derivan de la aplicación de los procesos de certificación y acreditación de programas académicos, en especial aquellos orientados a la formación de futuros docentes. Así mismo, reconocen en el PEI la carta de navegación para el cumplimiento de los objetivos misionales y de programas.

Tabla 2. Indicadores de la Dimensión Procesos de Acreditación

\begin{tabular}{lccc}
\hline \multicolumn{1}{c}{ Items } & \multicolumn{3}{c}{ Postura } \\
\cline { 2 - 4 } & $\begin{array}{c}\text { De } \\
\text { rechazo }\end{array}$ & Indiferente & $\begin{array}{c}\text { De } \\
\text { aceptación }\end{array}$ \\
\hline $\begin{array}{l}\text { Considera que los procesos de certificación y acreditación } \\
\text { de los programas de formación de docentes, deben } \\
\text { responder a la naturaleza y características de los } \\
\text { programas, sus modalidades y las diferencias territoriales } \\
\text { y poblacionales presentes en Colombia. }\end{array}$ & $3 \%$ & $1 \%$ & $96 \%$ \\
\hline $\begin{array}{l}\text { Los Proyectos Educativos Institucionales - PEl } \\
\text { contribuyen al desarrollo de los programas educativos. }\end{array}$ & $8 \%$ & $\mathbf{2 2 \%}$ & $\mathbf{7 0 \%}$ \\
\hline \multicolumn{1}{c}{ Porcentaje Promedio } & $\mathbf{6 \%}$ & $\mathbf{1 2 \%}$ & $\mathbf{8 3 \%}$ \\
\hline
\end{tabular}

En lo que respecta a la dimensión de Globalización, se pudo determinar que en promedio el $70 \%$ de los participantes poseen una postura de aceptación respecto a los ítems de esta dimensión, destacando que es necesario que las políticas del Estado actúen como alternativa de solución para superar tantas las necesidades sociales de la población y no que sean asumidas como una exigencia de organismos multilaterales con

Tabla 3. Indicadores de la Dimensión de Globalización

\begin{tabular}{|c|c|c|c|}
\hline \multirow[b]{2}{*}{ Items } & \multicolumn{3}{|c|}{ Postura } \\
\hline & $\begin{array}{c}D e \\
\text { rechazo }\end{array}$ & Indiferente & $\begin{array}{c}\text { De } \\
\text { aceptación }\end{array}$ \\
\hline $\begin{array}{l}\text { Considera que existe un afán gubernamental por cumplir las } \\
\text { exigencias de las agencias internacionales con respecto a la } \\
\text { globalización, mientras se descuidan los aspectos educativos, } \\
\text { superar la inequidad, la pobreza y el desastre ambiental. }\end{array}$ & $5 \%$ & $2 \%$ & $93 \%$ \\
\hline $\begin{array}{l}\text { El Estado Colombiano debe tomar las exigencias de } \\
\text { organismos multilaterales como herramientas de acción y no } \\
\text { como imposición que acarreen sanciones o implicaciones en el } \\
\text { sector económico. }\end{array}$ & $9 \%$ & $6 \%$ & $85 \%$ \\
\hline $\begin{array}{l}\text { El principal interés de los organismos como la OCDE, el Banco } \\
\text { Mundial, el Fondo Monetario Internacional, entre otros; es } \\
\text { asegurar procesos educativos que contribuyan al desarrollo } \\
\text { social de las naciones. }\end{array}$ & $48 \%$ & $21 \%$ & $31 \%$ \\
\hline Porcentaje Promedio & $21 \%$ & $10 \%$ & $70 \%$ \\
\hline
\end{tabular}


En cuanto a la dimensión de Procesos Formativos en promedio el $97 \%$ de los participantes manifestaron estar de acuerdo con las intensiones sugeridas en esta dimensión, resaltando la complementariedad que debe existir en el proceso de formación de todo licenciado, en lo que respecta a la conjugación de las competencias disciplinares, investigativas y pedagógicas, para así poder garantizar un docente que domine los saberes propios de la disciplina pero que realice un uso adecuado de los recursos pedagógicos para garantizar el entendimiento de los conceptos y que se apoye de la investigación como recurso para el desarrollo de diversas habilidades como el pensamiento crítico, el razonamiento, el planteamiento y ejecución de posibles alternativas de solución ante situaciones problema.

Tabla 4. Indicadores de la Dimensión Procesos Formativos

\begin{tabular}{lccc}
\hline \multicolumn{1}{c}{ Items } & \multicolumn{2}{c}{ Postura } \\
\cline { 2 - 3 } & $\begin{array}{l}\text { De } \\
\text { rechazo }\end{array}$ & Indiferente & De aceptación \\
\hline $\begin{array}{l}\text { Los programas de Licenciatura deben fomentar la } \\
\text { formación del saber pedagógico y del saber disciplinar } \\
\text { de forma armonizada. }\end{array}$ & $0 \%$ & $1 \%$ & $99 \%$ \\
\hline $\begin{array}{l}\text { La formación investigativa debe ser un factor } \\
\text { fundamental en los procesos de formación de maestros } \\
\text { en Colombia. }\end{array}$ & $3 \%$ & $\mathbf{2} \%$ & $\mathbf{9 5 \%}$ \\
\hline \multicolumn{1}{c}{$\quad$ Porcentaje Promedio } & $\mathbf{2 \%}$ & $\mathbf{2 \%}$ & $\mathbf{9 7 \%}$ \\
\hline
\end{tabular}

Finalmente, en la dimensión de Formación Docente se destaca como elementos importantes en opinión de los participantes la inconformidad que existe a nivel social y salarial por la falta de reconocimiento ante la labor que realizan los docentes, dada su importancia en el proceso de formación de futuras generaciones, es una labor que demanda de estar constantemente

Tabla 5. Indicadores de la Dimensión Formación Docente

\begin{tabular}{lccc}
\hline \multicolumn{1}{c}{ Items } & \multicolumn{2}{c}{ Postura } \\
\cline { 2 - 4 } & $\begin{array}{c}\text { De } \\
\text { rechazo }\end{array}$ & Indiferente & $\begin{array}{c}\text { De } \\
\text { aceptación }\end{array}$ \\
\hline $\begin{array}{l}\text { Los vacíos de formación de los docentes (herramientas de } \\
\text { las TIC y otros ámbitos) pueden ser subsanados mediante } \\
\text { procesos de actualización posteriores a la titulación. }\end{array}$ & $35 \%$ & $8 \%$ & $57 \%$ \\
\hline $\begin{array}{l}\text { La labor de los docentes es muy exigente, sin embargo, } \\
\text { se perciben que son profesionales de menor nivel, con } \\
\text { salarios bajos y poca exigencia académica. }\end{array}$ & $7 \%$ & $4 \%$ & $89 \%$ \\
\hline \multicolumn{1}{c}{ Porcentaje Promedio } & $\mathbf{2 1 \%}$ & $\mathbf{6 \%}$ & $\mathbf{7 3 \%}$ \\
\hline
\end{tabular}




\section{CONCLUSIONES}

El presente estudio genera varios espacios de reflexión que evidencian la necesidad de fortalecer y armonizar el saber pedagógico con el saber disciplinar y dar nuevas posibilidades a los maestros en formación para que interactúen con los contextos de práctica pedagógica de una forma más permanente y con mayor rigor educativo.

Sehacenecesarioquese realicen investigaciones donde se visibilicen las transformaciones en las prácticas pedagógicas y la calidad educativa derivadas de los cambios legislativos en los programas de formación de maestros que se han realizado en el país en los últimos años.

Los resultados mostrados en este texto ponen de manifiesto las inconformidades de maestros y directivos docentes con relación a la formulación de políticas públicas en temas de formación de maestros en Colombia, principalmente en los procesos de acreditación de alta calidad y de autonomía universitaria.

Dentro de las expectativas de los participantes del estudio resaltan la articulación del sistema escolar colombiano con los debates y transformaciones de los campos científicos y humanistas contemporáneos, así como con la diversidad territorial y poblacional del país.

\section{REFERENCIAS BIBLIOGRÁFICAS}

Arias Gómez, D. H., Díaz Flórez, O. C., Garzón Barragán, I., León Palencia, A. C., Rodríguez Ávila, S. P., \& Valbuena Ussa, É. O. (2018). Entre las exigencias de calidad y las condiciones de desigualdad: Formación inicial de profesores en Colombia. Universidad Pedagógica Nacional.

Báez Osorio, Miryam (2005). Las Escuelas Normales colombianas y la formación de maestros en el siglo XIX. Eccos Revista Científica, 7 (2), 427-450. Recuperado de: https:// www.redalyc.org/articulo.oa?id=715/71570210
Beltrán, R. R. (2018). La práctica pedagógica como herramienta para historiar la pedagogía en Colombia. Pedagogía y saberes, (49), 27-40. DOI: https://doi.org/10.17227/pys.num49-8168

Betancourt Serna, F., \& Tovar Torres, C. (2012). Documentos Inéditos para la Historia de la Educación en Colombia. Educación y Territorio, 2 (2), 135-139. Recuperdo de http://hdl.handle. net/11441/54019

Castro, J. O. (2000). Historia de la educación y la pedagogía. Una mirada a la configuración de un campo del saber. Henao Willes, M. Y Castro, Jo (Comp.) Estados del arte de la investigación en Educación y Pedagogía en Colombia I. Bogotá: ICFES, Colciencias, Sociedad Colombiana de Pedagogía, 235-278.

Cavadias, L. M. (2014). Importancia de la formación del profesorado y su impacto en el proceso educativo desde la primera infancia. Saber, ciencia y libertad, 9(1), 147156. Doi: https://doi.org/10.18041/2382-3240/ saber.2014v9n1.2003

Figueroa, Claudia (2006). Orígenes, formación y proyección de las Facultades de Educación en Colombia 1930 - 1954. Revista Historia de la Educación Latinoamericana, 8, 201-220. Recuperado de https://www.redalyc.org/articulo. oa? $\mathrm{id}=869 / 86900810$

Geles, Y. P. M., \& Ferreira, N. V. C. (2018). Escuelas normales de mujeres en Colombia (1903-1914). Cadernos De História DaEducação, 17(1), 260-274. https://doi.org/10.14393/chev17n1-2018-16. Doi: https://doi.org/10.14393/ che-v17n1-2018-16

Garcés, O. L. Z. (1994). Las escuelas normales en Colombia (durante las reformas de Francisco de Paula Santander y Mariano Ospina Rodríguez). Revista educación y pedagogía, (12-13), 263-278. 
Gamboa, A. (2016). Calidad de la Educación Superior: pretensiones y realidades institucionales. Bogotá, Colombia: Ecoe Ediciones.

George, D. M., \& Mallery, Y. P. (2003). SPSS for Windows step by step: A Simple Guide and Reference. 11.0 Update.

Herrera, M. C. (1993). Historia de la educación en Colombia. La República Liberal y la modernización de la educación: 19301946. Revista colombiana de educación, (26).

Montes, A. J., Romero, Z., \& Gamboa, A. (2017). La formación docente en el marco de la política de calidad de la Educación Básica en Colombia. Revista Espacios, 38(20).

Ministerio de Educación Nacional. (2013). Sistema Colombiano de Formación de Educadores y Lineamientos de Política. Bogotá, Colombia: Imprenta Nacional.

Navarrete-Cazales, Z. (2018). La carrera de Pedagogía en la Universidad Veracruzana. Historia fundacional y curricular 19542000. Perfiles educativos, 40(160), 47-63. Recuperado de http://www.scielo.org.mx/ scielo.php?script $=$ sci_arttext\&pid=S0185$26982018000200047 \&$ lng=es\&tlng=es.

República de Colombia. (1994). Ley 115. Por la cual se expide la Ley General de Educación. Diario Oficial 41214. Recuperado de https://www. mineducacion.gov.co/1759/articles-85906_ archivo_pdf.pdf

República de Colombia. (1998). Por el cual se establecen los requisitos de creación y funcionamiento de los programas académicos de pregrado y postgrado en Educación. Recuperado de https://www.mineducacion.gov. co/1759/w3-article-86202.html?_noredirect=1
República de Colombia. (2002). Por el cual se expide el Estatuto de Profesionalización Docente. Recuperado de https://www.mineducacion.gov. co/1621/articles-86102_archivo_pdf.pdf

República de Colombia. (2019). Por el cual se sustituye el Capítulo 2 y se suprime el Capítulo 7 del Título 3 de la Parte 5 del Libro 2 del Decreto 1075 de 2015 -Único Reglamentario del Sector Educación. Recuperado de https://www. mineducacion.gov.co/1759/w3-article-387348. html?_noredirect=1

Rátiva-Velandia, M. (2018). Escuela Normal Colombiana frente a las disposiciones legales. Tensiones Pedagógicas $y$ administrativas. Praxis, 14(1), 9-24. Doi: https:// doi.org/10.21676/23897856.2537 